\title{
Parameters of the preproduction series SiPMs for the CMS HCAL Phase I upgrade
}

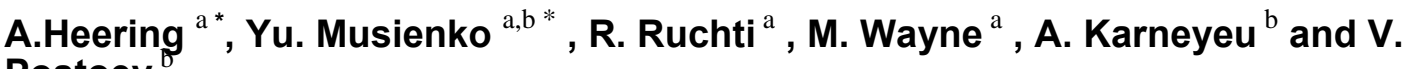 \\ Postoev \\ a. University of Notre Dame, Notre Dame, IN 46556, USA \\ b. Instutute for Nuclear Research RAS, pr. 60-letiya Oktyabrya 7a, 117312 Moscow, Russia \\ E-mail: Adriaan.Heering@cern.ch \\ E-mail: Iouri.Musienko@cern.ch
}

\begin{abstract}
In 2012 the HCAL SiPM photo sensor upgrade was approved for the increased luminosity $\left(5^{*} 10^{34}\right)$ of SLHC. The upgrade will replace the current hybrid photodiodes (HPDs) with multi-pixel Geiger-mode avalanche photodiodes, also known as silicon photomultipliers (SiPMs). A key aspect of the upgrade to SiPMs is to add longitudinal segmentation and improve signal to noise to compensate for scintillator radiation damage. After 5 years of R\&D with multiple companies we developed custom large dynamic range SiPMs with large PDE and small ENC. To ensure good mechanical alignment and easy handling of the large number of production channels $(>20000)$ we have developed a custom ceramic package with a very thin $0.3 \mathrm{~mm}$ quartz window with Kyocera. Each package holds 8 channels of SiPMs.

Here we report on the final SiPM parameters of the 2014 preproduction run from Hamamatsu (HPK) who has produced a series of 175 arrays with a total of 1400 SiPMs. An overview of our QA results and measurements of the photon detection efficiency, spectral response, crosstalk and cell recovery time will be discussed.
\end{abstract}

KEYWORDS: Silicon photomultiplier, MPPC, GAPD, CMS, HCAL.

PACS: 85.60.Bt, 85.60.Ha

* Corresponding authors. 


\section{Introduction.}

The CMS Barrel (HB) and Endcap (HE) Hadron Calorimeters are scintillator sampling calorimeters with embedded wavelength shifting fibers (WLS) in scintillator tiles. The fibers from the sampling layers are ganged together to form towers whose light is detected by photosensors. The photo-sensors that are currently used are HPDs. In 2012 the HCAL SiPM photo sensor upgrade was approved for the increased luminosity $\left(5^{*} 10^{34}\right)$ of SLHC. The technical design report has the details of the complete upgrade [1]. The replacement of the 19 channel HPD by 48 channels of SiPMs will give three times more depth segmentation. The higher gain and PDE can give almost 20x better S/N, improving background rejection and energy resolution. Because the anticipated radiation damage in the scintillators is nonlinear with the depth, the extra segmentations will give us the opportunity to do depth dependent radiation damage compensation.

The SiPMs for the CMS HCAL upgrade must operate in a very hostile SLHC radiation environment. We expect a maximum total dose of $10^{12} \mathrm{n} / \mathrm{cm}^{2}$ for an integrated luminosity of $3000 \mathrm{fb}^{-1}$ (Recent studies on radiation hardness of the devices discussed are beyond the scope of this paper but could be found in [2-4]). The SiPMs should have good linearity for a wide range of $1 \mathrm{GeV}$ to $10 \mathrm{TeV}$ of scintillating signals and excellent reliability. Our R\&D on large dynamic range SiPMs in collaboration with Hamamatsu and other companies started in 2010. The final configuration is a custom packaged array of $8 \mathrm{SiPMs}$. Figure 1 shows the arrays of 15 micron cell devices in 2 sizes; $2.8 \mathrm{~mm}$ and $3.3 \mathrm{~mm}$ diameter with reps. 27500 and 38500 cells. The 2.8 $\mathrm{mm}$ will read out a sum of 4 fibers and the $3.3 \mathrm{~mm}$ will read out a sum of 7 fibers coming from the HCAL scintillators. Hamamatsu (HPK) was selected to produce a preproduction series of 175 Arrays. The main characteristics of the SiPMs arrays will be discussed.

\section{Choice of package with quartz window.}

A custom package holding 8 SiPMs was developed with Kyocera (Germany). The package has two alignment pin holes to align the fiber bundles from the detector to the SiPMs.

Because scattering neutrons in the CMS detector interact with epoxy giving large fake trigger pulses, a thin $0.3 \mathrm{~mm}$ quartz window with antireflective coating with cut off at $420 \mathrm{~nm}$ was used instead of epoxy. By using an AmBe neutron source, we measured the noise rate vs. electronic threshold. Fig 2 shows the neutron interaction probability vs different media on top of the array. To simulate the presence of the fibers from the calorimeter on top of the package, a light mixer ( $2 \mathrm{~cm}$ long fiber with a diameter of $3 \mathrm{~mm}$ ) was placed on top of the package in the setup. Based on the data we chose, in addition to a quartz window, to use an antireflective coating with a cut off of $420 \mathrm{~nm}$ to suppress any residual Cherenkov light produced in the incoming fibers or quartz window itself. 


\section{Quality Assurance measurement results.}

\subsection{Dark current and response to LED vs. bias voltage}

For the quality assurance of many arrays, an 80 channel test setup was created allowing the measurement of I-V characteristics of 10 arrays at a time. The setup uses an 80 channel Keithley 7001 switch box in combination with a Keithley 6487 pico ammeter. A $520 \mathrm{~nm}$ LED was placed in the dark box to uniformly illuminate all 80 channels to measure the gain x PDE. Fig. $3 \mathrm{a}$ and $3 \mathrm{~b}$ show the dark current vs bias voltage of all 984 channels of the $2.8 \mathrm{~mm}$ arrays and 416 channels of the $3.3 \mathrm{~mm}$ arrays respectively at $23^{\circ} \mathrm{C}$. Fig. $3 \mathrm{c}$ and $3 \mathrm{~d}$ show the current of the same arrays under uniform LED illumination plotted vs over-voltage. We found that all channels except for one can reach a breakdown voltage of 4 volt. We also see that some channels in fig $3 \mathrm{a}$ and $3 \mathrm{~b}$ show a higher surface current (current below breakdown $>10 \mathrm{nA}$ ). We verified that the currents are stable after 4 weeks of operation at $70^{\circ} \mathrm{C}$ and temperature cycling between $-10^{\circ} \mathrm{C}$ and $50^{\circ} \mathrm{C}$ for up to one week.

From fig $3 \mathrm{c}$ and $3 \mathrm{~d}$ we can also conclude that we have very uniform gain and PDE vs over -voltage for all 1400 channels.

\subsection{Photo detection efficiency and crosstalk}

The Y11 WLS fibers in the CMS HCAL have an emission peak of $515 \mathrm{~nm}$. Fig. 4 shows the PDE at $515 \mathrm{~nm}$ and the crosstalk vs. over-voltage. HPK uses a transparent MFQR (Metal Film Quenching Resistor) to deliver high PDE for the small cell pitch devices of $15 \mathrm{x} 15 \mu \mathrm{m}$. Because the HCAL has a limited internal sampling resolution $100 \% / \sqrt{ } \mathrm{E}$ and a very low light yield per MIP, a trade of between PDE and Crosstalk is made by not using trench technology. Fig. 5 shows the spectral response vs wavelength. The setup and the experimental techniques used in these measurements are described in detail in [5]. The cut off due to the antireflective coating is clearly visible.

\subsection{Response to fast laser and recovery time}

The Y11 WLS fibers have a decay time of $11 \mathrm{~ns}$. To reduce the influence of the SiPMs on the total time response to a minimum, a specification of recovery time was set to $10 \mathrm{~ns}$. In order to accomplish this we specified a maximum value of $1.2 \mathrm{M} \Omega$ quenching resistor $(\mathrm{Rq})$ for the preproduction series. In addition HPK reduced the series resistance (Rs) present in larger area devices using segmentation. Fig. 6a shows the capacitance vs bias voltage of the 2.8 and 3.3 $\mathrm{mm}$ devices. We found 220 and $310 \mathrm{pF}$ at operating voltage $(\sim 70 \mathrm{~V})$ respectively. The forward resistance (Rf) can be found by measuring the forward I-V of the SiPMs, where Rf is equal to $\mathrm{Rq} /$ number of cells + Rs. Fig. $6 \mathrm{~b}$ shows Rf for 8 channels of a $2.8 \mathrm{~mm}$ array and 8 channels of a $3.3 \mathrm{~mm}$ array. We found $31.5 \pm 1.5 \Omega$ and $24.7 \pm 1.3 \Omega$ (at $1.4 \mathrm{~V}$ ) respectively. Fig $6 \mathrm{c}$ and $6 \mathrm{~d}$ show the response of the $2.8 \mathrm{~mm}$ and $3.3 \mathrm{~mm}$ arrays to a 20 ps laser diode pulse into $15 \Omega$ input resistance. From the pulse shape we found the recovery time of 7 and $8 \mathrm{~ns}$ respectively. 


\section{Conclusion}

Recent developments of small cell SiPMs make it possible to use them in large dynamic range detectors like the CMS HCAL. Enormous progress has been made in the PDE with these smaller cell devices. Other benefits of the small cell devices are lower gain, faster recovery time, radiation hardness and lower noise. After evaluation of many manufactures we did a preproduction run of $1400 \mathrm{SiPMs}$ with HPK. The most important results from an evaluation of this preproduction run were presented. We found good uniformity of gain, PDE and pulse shape. This uniform behavior could be especially valuable in the final calibration of the CMS HCAL detector.

\section{Acknowledgments}

The research leading to these results has received funding from the European Commission under the FP7 Research Infrastructures project AIDA, grant agreement no. 262025. This work was supported by the U.S. National Science Foundation and by the Russian Ministry of Education (Russian state grant RFMEFI61014X0004).

\section{References}

1. The CMS TDR for the phase I upgrade of the HCAL, CERN-LHCC-2012-015.

2. Y. Musienko et al., Nucl. Instr. and Meth. A 610 (2009) 87.

3. A. Heering et al.,Radiation Damage studies on SiPMs for calorimertry at the super LHC, 2007 IEEE Nuclear Science Symposium Conference Record, Vol. 2, 1523-1526 (2008).

4. Y. Musienko, A. Heering, R. Ruchti et al., Nucl. Instr. and Meth. A 787 (2015) 319.

5. Y. Musienko, S. Reucroft, J. Swain, Nucl. Instr. and Meth. A 567 (2006) 57.

Figure captions:

Fig. 1. Custom CMS HCAL 8 channel array with $2.8 \mathrm{~mm}$ and $3.3 \mathrm{~mm}$ diameter SiPMs.

Fig. 2. Neutron interaction probability using epoxy vs. a quartz window .

Fig. 3. Dark current and LED response vs bias voltage at $\mathrm{T}=23^{\circ} \mathrm{C}$. a) Dark current of all $1232.8 \mathrm{~mm}$ arrays. b) Dark current of all $523.3 \mathrm{~mm}$ arrays. c) LED response of all $2.8 \mathrm{~mm}$ arrays vs. over- voltage d) LED response of all arrays $3.3 \mathrm{~mm}$ vs. over-voltage

Fig. 4. PDE and crosstalk vs. over-voltage.

Fig. 5. Spectral response at 4 volt over-voltage.

Fig. 6. a) Capacitance vs. bias voltage of a $2.8 \mathrm{~mm}$ and $3.3 \mathrm{~mm}$ diameter SiPM. b) Forward resistance of a $2.8 \mathrm{~mm}$ and $3.3 \mathrm{~mm}$ diameter SiPM. c) Pulse shape of $2.8 \mathrm{~mm}$ diameter SiPM into 15 ohms. d) Pulse shape of $3.3 \mathrm{~mm}$ diameter SiPM into 15 ohms. 

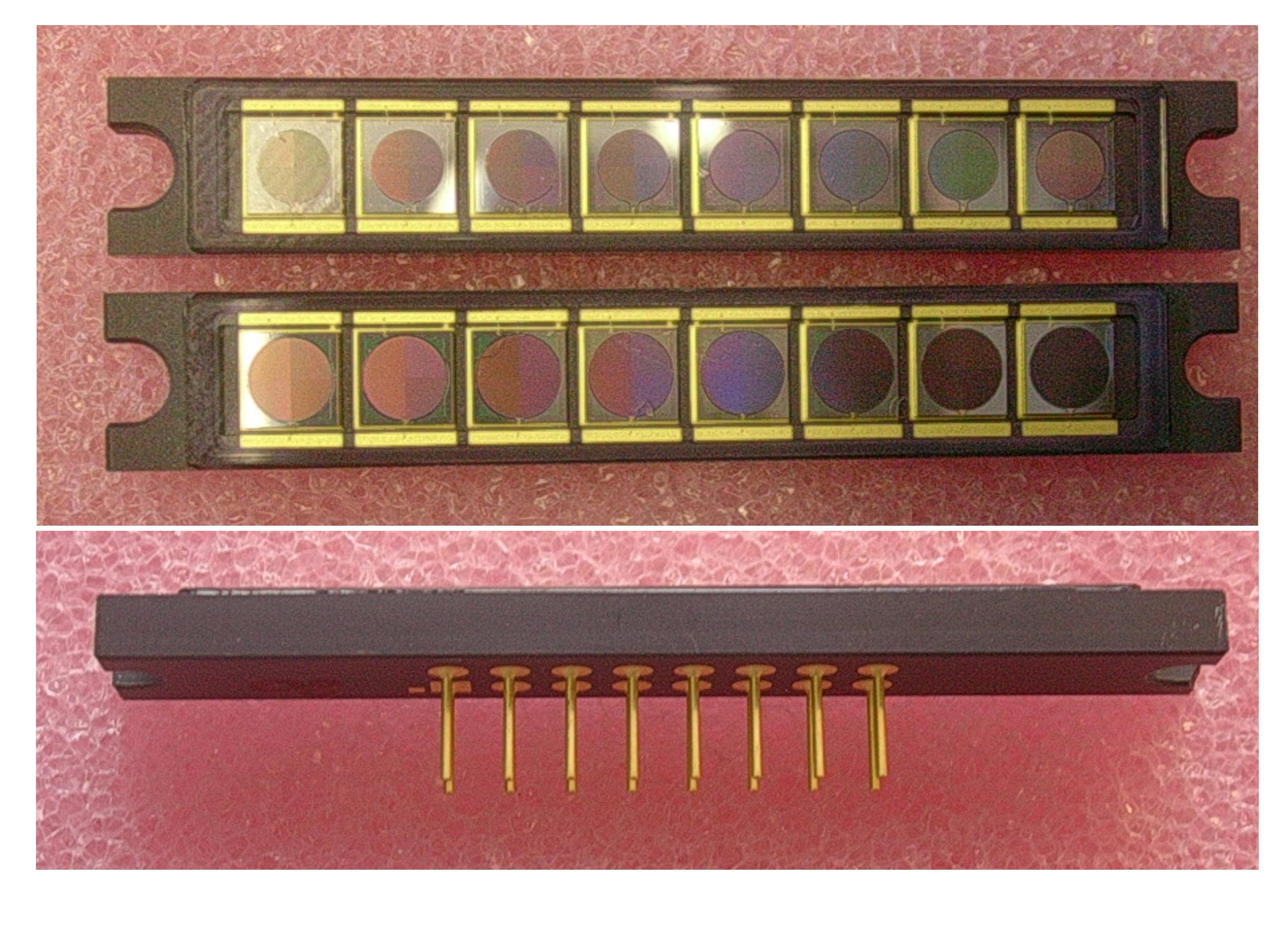


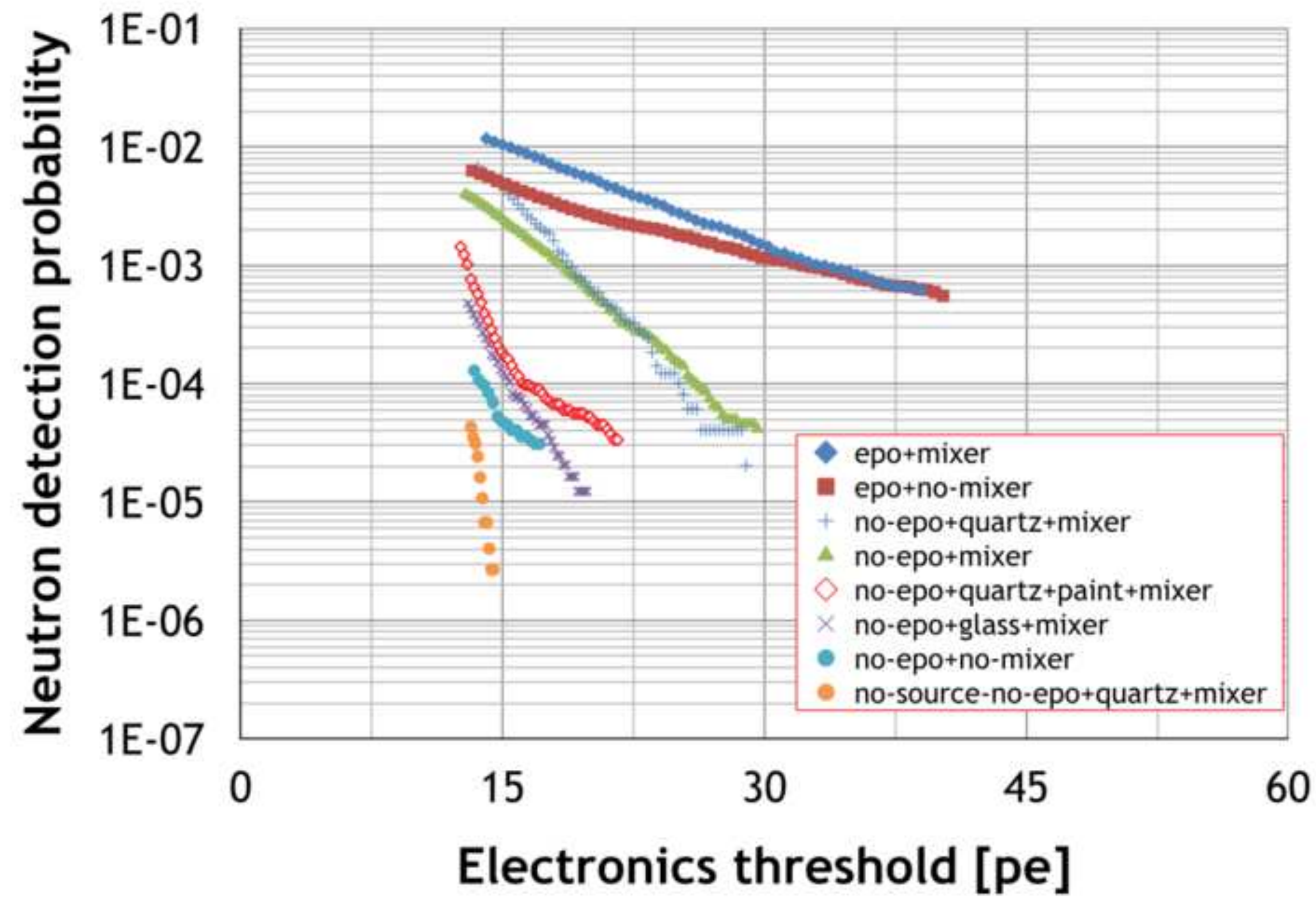



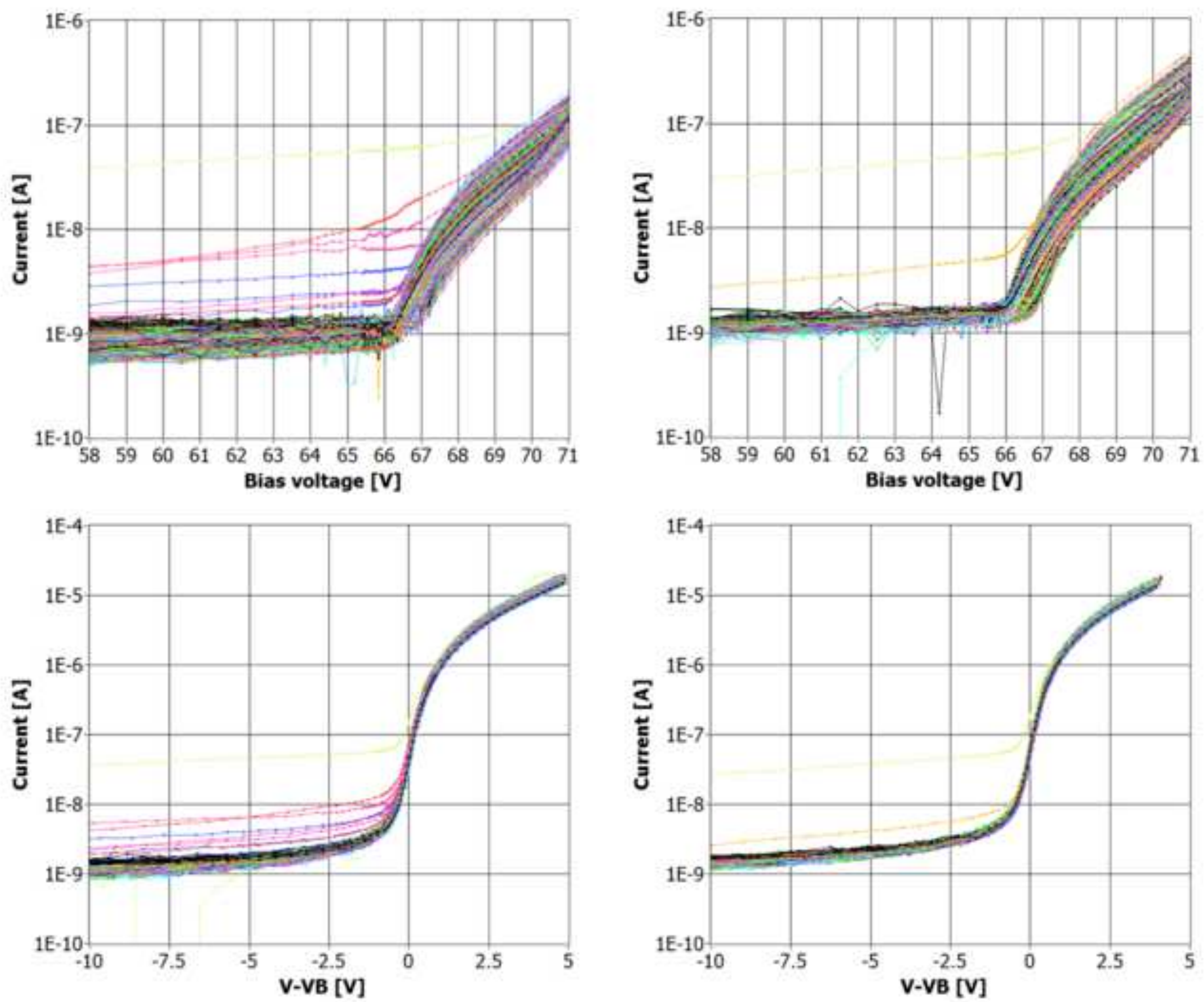

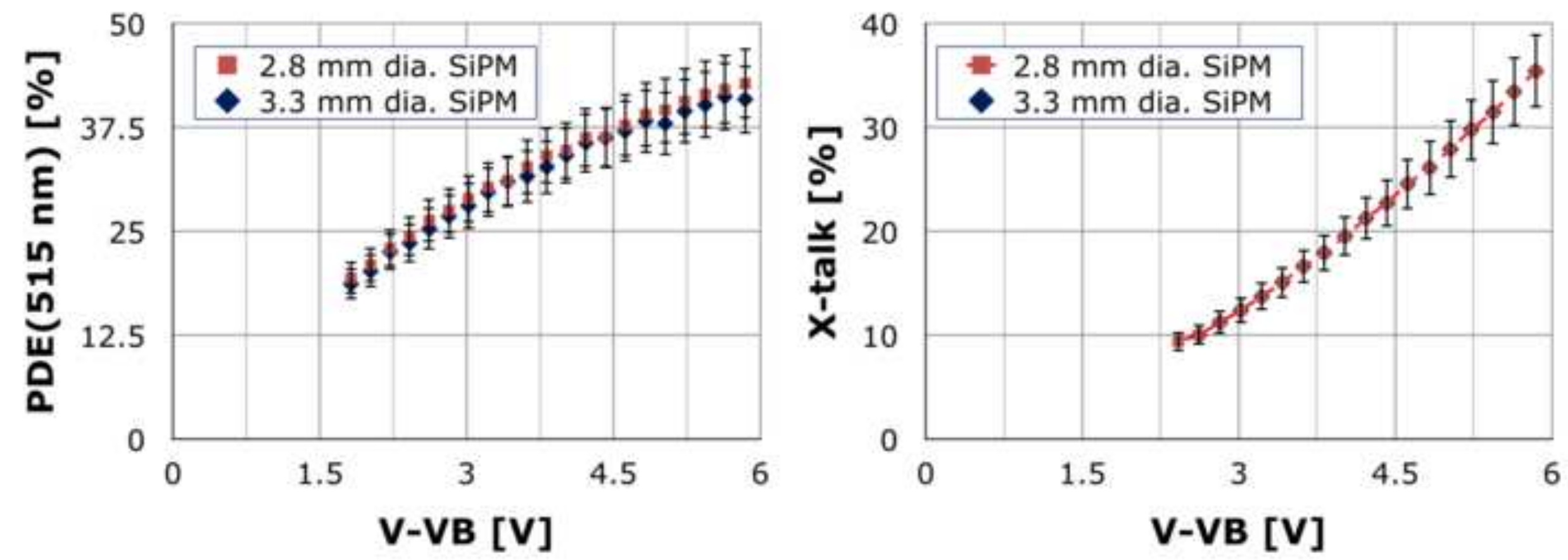


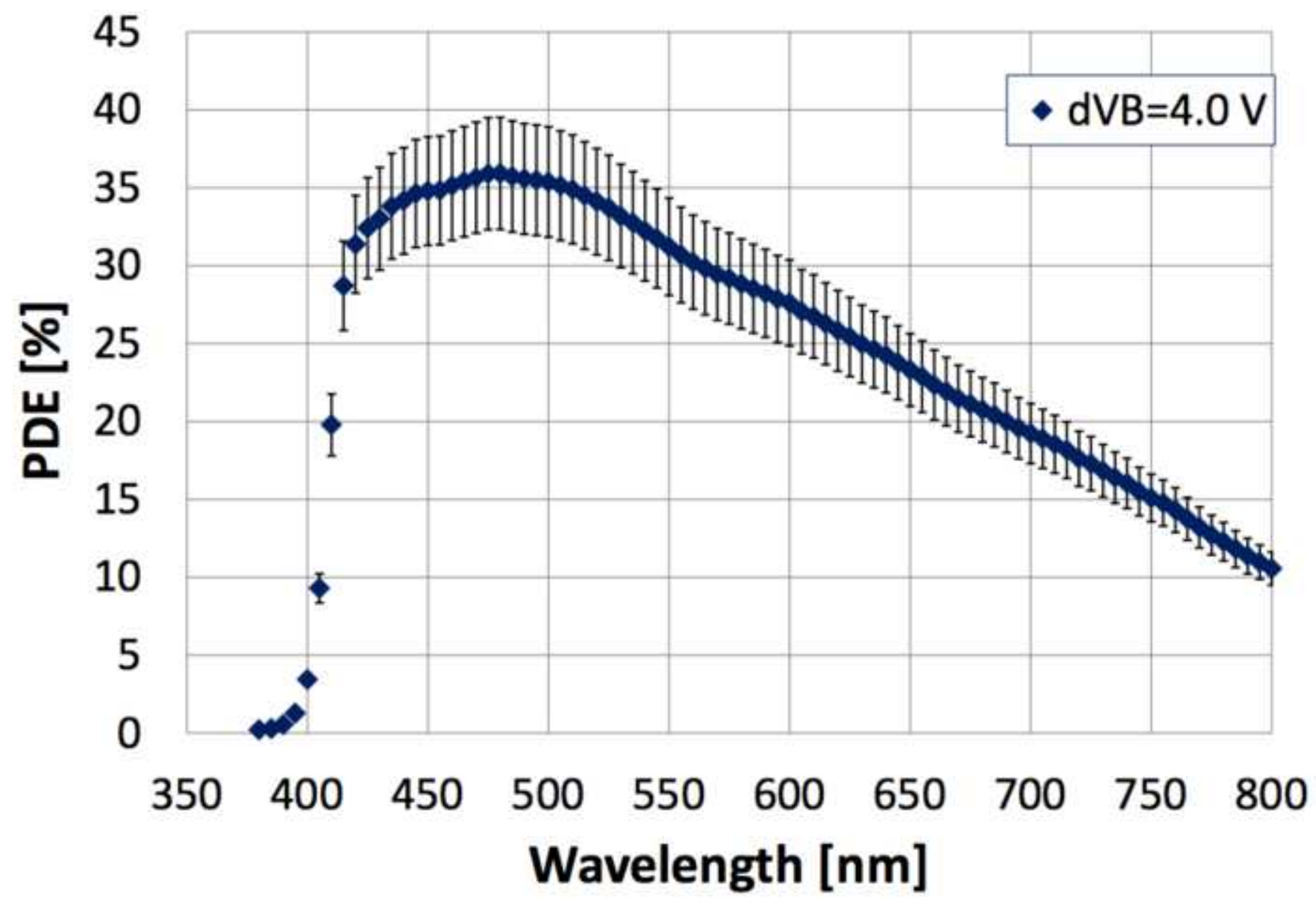



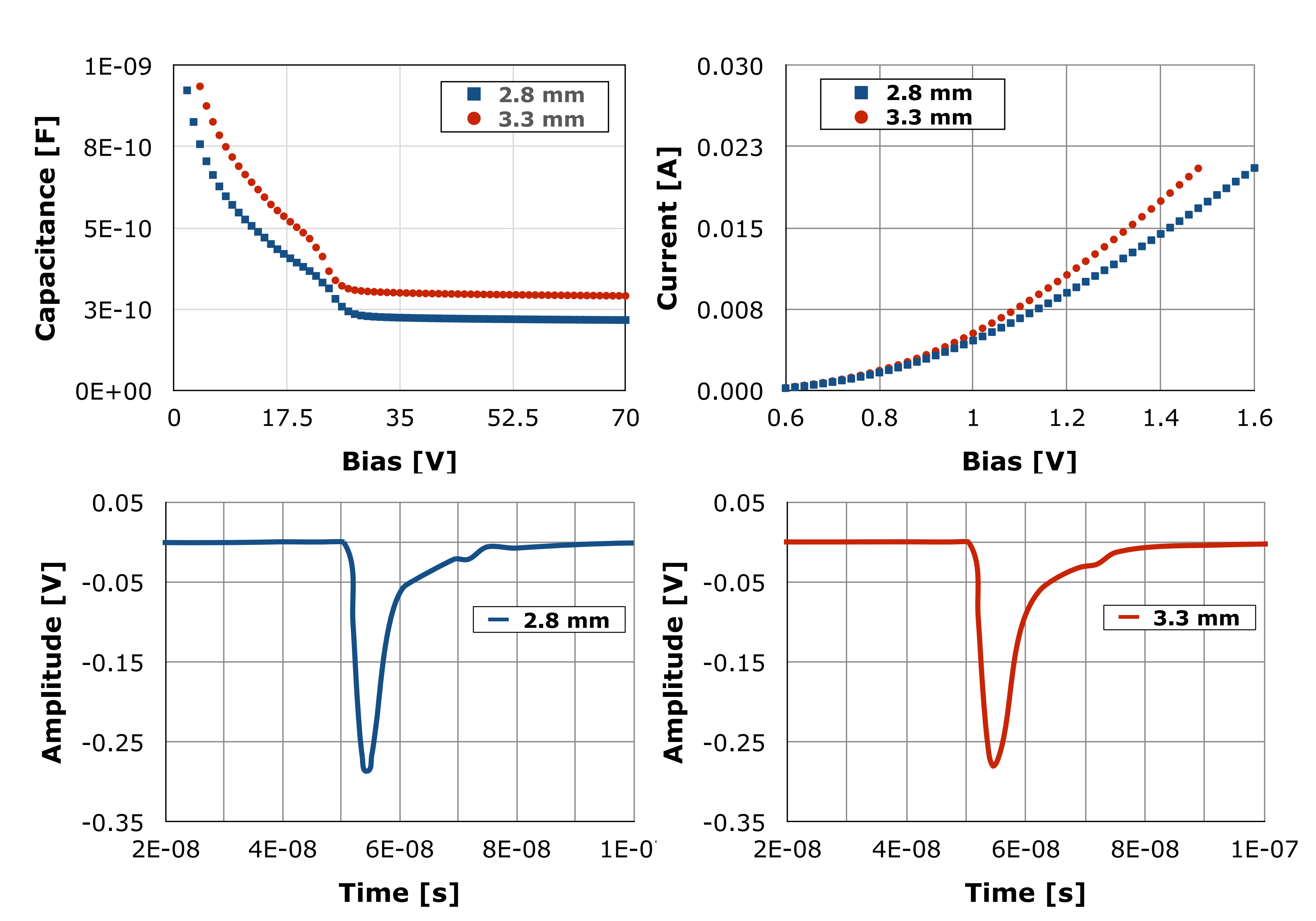